\title{
Laparoscopic Reconstruction of Pathologies Involving Solitary/ Predominantly Solitary Functioning Kidney- Feasibility, Safety and Outcome Analysis
}

Das K ${ }^{1 *}$, Abraham GP1,2, Siddiaiah AT1, Abraham $\mathrm{JJ}^{1}$, Ramaswami $\mathrm{K}^{2}$, Thampan $\mathrm{OS}^{2}$ and George $\mathbf{P D}^{3}$

${ }^{1}$ Lakeshore Hospital, Kochi, India

${ }^{2}$ PVS Memorial Hospital, Kochi, India

${ }^{3} \mathrm{AIMS}$, Kochi, India

*Corresponding author: Krishanu Das, Plot Senior Specialist Urology, Lakeshore Hospital, Kochi, India

Received: March 20, 2017; Accepted: June 15, 2017; Published: July 06, 2017

\begin{abstract}
Purpose: To evaluate the feasibility, safety and outcome of laparoscopic reconstruction of various urological pathologies in patients with Solitary Kidney (SK) or predominantly Solitary Functioning Kidney (PSK) (contralateral unit glomerular filtration rate $<10$ millilitres $/$ minute $\left./ \mathrm{m}^{2}\right)$.

Materials and Methods: Data of patients operated between January 2005 and December 2012 for similar scenarios was evaluated retrospectively. Preoperative imaging included Ultrasound (USG), Intravenous Pyelogram (IVP), CT or Magnetic Resonance Urogram (CTU or MRU), Diuretic Renogram (DR), Retrograde Urogram (RGU), Voiding Cystourethrogram (VCUG). Operative and postoperative profile was recorded. Follow-ups were scheduled 3 monthly. CTU or MRU and DR were repeated at 1 year post procedure. No intervention during intervening period (ureteral stent, nephrostomy or redo surgery) and improvement in clinical and radiological parameters was considered as successful outcome.
\end{abstract}

Results: Ten patients underwentlaparoscopic reconstruction-3 dismembered pyeloplasty (intrinsic pelviureteric dysfunction), 2 ureteroureterostomy (midureteric stricture), 1 Boari flap ureteroneocystostomy (long lower ureteral stricture), 1 tailoring and non-refluxing reimplantation (obstructive megaureter), and 3 non-refluxing ureteric reimplantation (grade IV vesicoureteric reflux). 5 patients revealed SK and 5 patients PFSK. Mean operative time was 160.5 minutes, mean blood loss was $52.5 \mathrm{ml}$ and mean hospital stay was $100.2 \mathrm{~h}$. There were no significant intraoperative and postoperative complications. Mean follow-up duration was 22 months. Significant improvement was noticed in last follow-up GFR (p 0.00, Paired t test).

Conclusion: Reconstruction of urological pathologies in SK or PSK through laparoscopic approach is feasible and safe. Satisfactory outcome can be achieved preserving the goals of minimally invasive access.

Keywords: Laparoscopy; Pyeloplasty; Pelviuretric junction obstruction; Kidney; Postoperative complications; Ureteral obstruction; Reconstruction

\section{Introduction}

Since its inception laparoscopic surgery has become an integral part of almost all urological ablative procedures [1]. Contrastingly in the field of reconstructive urology, the adoption of this approach was considerably delayed, probably due to the technical concerns [2]. Refinements in technique and increasing familiarity with the approach have resulted in more liberal application of this approach in the field of reconstructive urology in the present decade [3]. Laparoscopic pyeloplasty currently ranks as the most commonly performed urological reconstruction. The reported long term outcomes following this approach have been at par with the conventional open approach and this is currently considered the standard of care approach for pelviureteric rehabilitation [4,5]. In recent times, other reconstructive surgeries like ureteroureterostomy, Boari flap and ureteric reimplantation have also been increasingly performed via laparoscopic or robotic assisted laparoscopic access.
However there is paucity of data in the literature about laparoscopic reconstructions for various urological pathologies in patients with solitary or predominantly solitary functioning kidney. Although laparoscopic approach is not absolutely contraindicated in these scenarios, in view of circumstantial challenges probably incisional approach remains the favored modality in such circumstances.

We narrate our experience in laparoscopic reconstruction of various urological pathologies in solitary or predominantly solitary functioning kidney and analyse the feasibility, safety, morbidity profile and renal function outcome.

\section{Materials and Methods}

Patients with SK/PSFK who underwent reconstructive procedures for various urological pathologies between January 2005 and December 2012 were included in the patient cohort. Permission was obtained from institutional review board. All procedures
Austin J Clin Case Rep - Volume 4 Issue 2 - 2017 ISSN : 2381-912X | www.austinpublishing group.com Das et al. (C) All rights are reserved
Citation: Das K, Abraham GP, Siddiaiah AT, Abraham JJ, Ramaswami K, Thampan OS, et al. Laparoscopic Reconstruction of Pathologies Involving Solitary/Predominantly Solitary Functioning Kidney-Feasibility, Safety and Outcome Analysis. Austin J Clin Case Rep. 2017; 4(2): 1120. 
were performed by a single surgeon proficient in laparoscopic surgeries (GPA) and were conducted in two centers. Pre procedure evaluation included assessment of presenting complaints, previous clinical profile and previous surgical intervention. Routine blood investigations and renal function tests were carried out. All patients underwent preliminary ultrasound screen. Patients with suspected PUJ obstruction, ureteric stenosis or obstructive megaureter underwent CTU or MRU (in patients with altered renal profile at presentation), DR and RGU. Ureteric stenosis were classified as distal (distal to sacroiliac joint to vesicoureteric junction), middle (over the sacroiliac joint) or proximal (above the sacroiliac joint to pelviureteric junction) based on location of the pathology. Patients with a history suggestive of Vesicoureteric Reflux (VUR) were evaluated with Voiding Cystourethrogram (VCUG) and DR. In lower ureteric and vesicoureteric pathologies, cystoscopy was performed to rule out coexistent bladder pathologies. Patients revealing gross pelvicaliceal dilatation and altered renal profile at presentation underwent urinary diversion (Double J ureteral stent or percutaneous nephrostomy) prior to definitive correction. All laparoscopic procedures were undertaken via transperitoneal access.

\section{Operative exercise}

Laparoscopic pyeloplasty: A dismembered pyeloplasty was performed in all scenarios. If tension was apprehensed during pelviureteric approximation, the entire renal unit was mobilized following the intra-Gerotas' fascia plane and displaced caudally. Pelviureteric anastomosis was undertaken employing interrupted sutures of 4-0 polyglactin. Any additional closure of the excised redundant pelvis was conducted in a continuous manner. Ureteral stent was inserted antegrade prior to anastomotic completion.

Laparoscopic ureteroureterostomy: Ureteric mobilisation was performed and pathological segment delineated. Dismemberment, exclusion of the culprit segment, spatulation of opposite ends and tension free reunion remained the subsequent exercises. Meticulous attention was paid to preservation of periureteral vascularity and optimum spatulation was conducted to ensure a wide anastomosis. A Double J ureteral stent was inserted in antegrade fashion and interposed across the anastomosis.

Laparoscopic boari flap: Our technique of laparoscopic Boari flap has been previously demonstrated [6]. Meticulous attention was paid to preserve ureteral and flap vascularity. Ureterovesical anastomosis and flap tubularisation was conducted with 3-0 polyglactin suture and bladder closure with 2-0 polyglactin suture.

Laparoscopic antireflux surgery: Our technique of laparoscopic antireflux surgery has been previously demonstrated [7]. Care was taken to preserve the vas deferens that lies in the vicinity of vesicoureteric junction. Detrusorraphy was conducted using interrupted 3-0 polyglactin sutures.

Laparoscopic ureteral tailoring: Our technique of laparoscopic ureteric tailoring has been previously demonstrated [8]. The ureter was dismembered immediately proximal to the pathological segment and all tortuosities straightened. The ease of approximation of the dismembered ureter to the bladder was ascertained. Thereafter, the lower 5 centimeters of the straightened ureter was tailored. The ureteral circumference was reduced to a size that just accommodates

Table 1: Demographic data.
\begin{tabular}{|c|c|}
\hline Variable & Value \\
\hline Mean (SD,Range) & $29(20.51,3-650)$ \\
\hline Age, years & $21.1(3.32,15.2-24.2)$ \\
\hline BMI, Kg/m² & $1.78(1.08,0.6-3.6)$ \\
\hline Preoperative Serum creatinine, mg/dl & $28.7(4.64,22-35)$ \\
\hline Preoperative GFR, ml/min & 5 \\
\hline Solitary Kidney & 5 \\
\hline PSFK & 3 \\
\hline Culprit Pathology & 2 \\
\hline Intrinsic pelviureteric obstruction & 1 \\
\hline Midureteric stricture & 1 \\
\hline Long lower ureteral stricture & 3 \\
\hline Obstructive megaureter & \\
\hline Pediatric vesicoureteric reflux & \\
\hline
\end{tabular}

a 6 F ureteral stent. Ureteroneocystostomy was performed using 3-0 polyglactin followed by extravesical antireflux creation using 3-0 polyglactin.

\section{Follow-up}

Operative and postoperative details were recorded. Renal profile was assessed on first postoperative day and then at periodic intervals. Ureteral stent was removed 6 weeks post procedure. The followup protocol included 3 monthly revisits with evaluation of clinical parameters, renal profile and ultrasound. CTU or MRU and DR were repeated at 1 year post procedure.

\section{Outcome analysis}

No intervention during the follow-up period (ureteral stent, nephrostomy or redo surgery), stabilization or improvement in renal profile, stabilization or improvement in hydronephrosis and drainage parameters at 1 year was considered a successful outcome. Statistical interpretation.

Statistical analysis was done by using SAS software 9.2 version. A $p$ value $<0.05$ was inferred as statistically significant.

\section{Results}

Demographic details are projected in Table 1. At time of definitive reconstruction, 5 patients revealed SK and 5 patients revealed PSK. The patient with lower ureteric stricture underwent preoperative nephrostomy drainage 3 months prior to definitive correction. In all other cases definitive procedure was undertaken without any temporary urinary diversion. All 10 patients underwent laparoscopic reconstruction successfully-3 dismembered pyeloplasty (intrinsic pelviureteric dysfunction), 2 ureteroureterostomy (midureteric stricture), 1 Boari flap ureteroneocystostomy (long lower ureteral stricture) and 1 tailoring and non-refluxing reimplantation (obstructive megaureter) and 3 non-refluxing ureteric reimplantation (pediatric vesicoureteric reflux, Grade III-IV). The operative, postoperative and follow-up profile is depicted in Table 2. There were no remarkable intraoperative and postoperative events. A significant elevation was appraised in first postoperative day creatinine from preprocedure values ( $\mathrm{p} 0.00$ ). Till last follow-up, all patients reported 
Table 2: Operative and postoperative details.

\begin{tabular}{|c|c|}
\hline Variable & Mean, (SD, Range) \\
\hline Operative duration, minutes & $160.5(30.41,100-200)$ \\
\hline Blood loss, $\mathrm{ml}$ & $52.5(18.45,25-75)$ \\
\hline Oral intake, hours & $16.4(4.6,12-26)$ \\
\hline Postop day 1 serum creatinine, $\mathrm{mg} / \mathrm{ml}$ & $2.05(1.16,0.8-4)$ \\
\hline Time to drain removal, hours & $52.8(18.26,26-72)$ \\
\hline Hospital stay, hours & $100.2(10.81,90-120)$ \\
\hline Follow up duration, months & $22(13.74,6-54)$ \\
\hline Last follow up serum creatinine, $\mathrm{mg} / \mathrm{ml}$ & $1.29(0.69,0.5-2.8)$ \\
\hline Last follow up GFR, ml/min & $39.1(4.99,28-45)$ \\
\hline
\end{tabular}

an improved clinical profile. 9 patients completed 1 year followup. No patients required any intervention during the intervening period. Figure 1 potrays the comparative analysis of preoperative and postoperative creatinine and GFR. Last follow-up creatinine was statistically equitable to the preprocedure levels ( $\mathrm{p}=0.07, \mathrm{t}$ test). A significant improvement was remarked in last follow-up GFR in comparison to the preoperative profile ( $\mathrm{p}=0.001, \mathrm{t}$ test).

\section{Discussion}

Several issues need to be addressed to ensure satisfactory reconstruction of pelviureteric, ureteric or vesicoureteric pathologies. In patients with extensive fibrosis, identification and isolation of the pathological segment remains a major challenge and familiarity with laparoscopic anatomy is mandatory. Preservation of optimum vascular inflow to the salvageable segments of the ureter is also of utmost importance. This goal may be achieved by meticulous preservation of the periureteral adventitia during ureteric mobilisation. In pelviureteric and midureteric pathologies necessitating exclusion of the pathological segment, optimum length of ureter should be preserved to ensure a tension free reunion of the healthy margins. If anastomotic tension is apprehensed, sufficient mobilization of the dismembered segments should be conducted. Reconstructions in long segment lower ureteric pathologies mandate creation of bladder flaps and attention should be directed at achieving satisfactory flap dimensions as well as maintaining flap vascularity. In obstructive megaureter, suboptimal adhesiolysis, straightening or downsizing leads to redundancy and hinders satisfactory attainment of ureteral tone and peristalsis, whereas over vigorous ureteric mobilization may jeopardize ureteral vascularity and increase the propensity of ureteric stricture. Apart from proficiency in tissue handling and reasonable experience in dealing with these pathologies, the operator needs to be versed with intracorporeal suturing exercises. In the setting of solitary kidney, these complexities may be further accentuated by the presence of deranged renal function preprocedure, effect of prolonged pneumoperitoneum on renal function and narrow margin for error. There is sufficient data in current literature to support the laparoscopic reconstruction of various pelviureteric and ureteric pathologies, albeit in bilaterally functioning units. Familiarity with similar exercises in bilaterally functioning units motivated the operator to undertake these reconstructions. Pelviureteric junction obstruction has been addressed by incisional, laparoscopic or endourologic approaches. The till date reported outcomes following endourological exercises has not been impressive $[9,10]$. Contrastingly many large series have established the efficacy of laparoscopic pyeloplasty $[2,11,12]$. However these citations seldom reflect the experiences in solitary or predominantly solitary functioning units. Justin M Albani, et al. employed different modalities of treatment for UPJO in a cohort of patients with solitary renal unit [13]. 15 patients underwent different surgical approaches (laparosopic pyeloplasty-3 cases, endopyelotomy-3 cases and open pyeloplasty-9 cases). A statistically significant improvement in renal function was obtained in all groups. However this study does not elaborate the preoperative renal profile status of all sufferers. In a case report, Shah, et al. have also reported safety and feasibility of laparoscopic pyeloplasty in solitary kidney [14]. In our cohort of patients with UPJO, one patient presented with deranged renal profile and 2 patients demonstrated normal renal profile. All patients revealed normal renal profile till last revisit. An appreciable improvement in follow-up imaging and diuretic renogram paramaters was observed universally.

Restorative options for benign ureteric stricture are dictated by the location and length of stricture. Conventional approaches include ureteroureterostomy, ureteroneocystostomy, boari flap reimplantation or incorporation of intestinal segments. Successful construction of laparoscopic Boari flap reconstruction was first published by Kavoussi and associates from John Hopkins centre in 2001 [15]. 3 patients with long lower ureteric stricture underwent boari flap reconstruction through laparoscopic approach. All

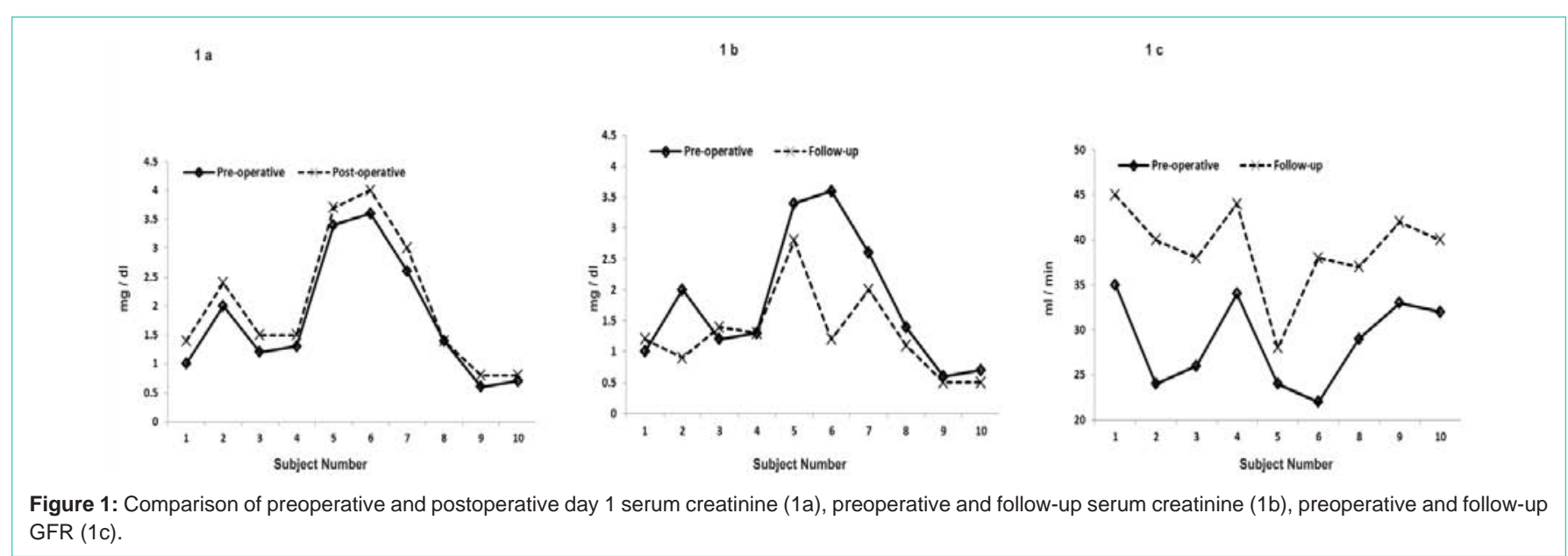




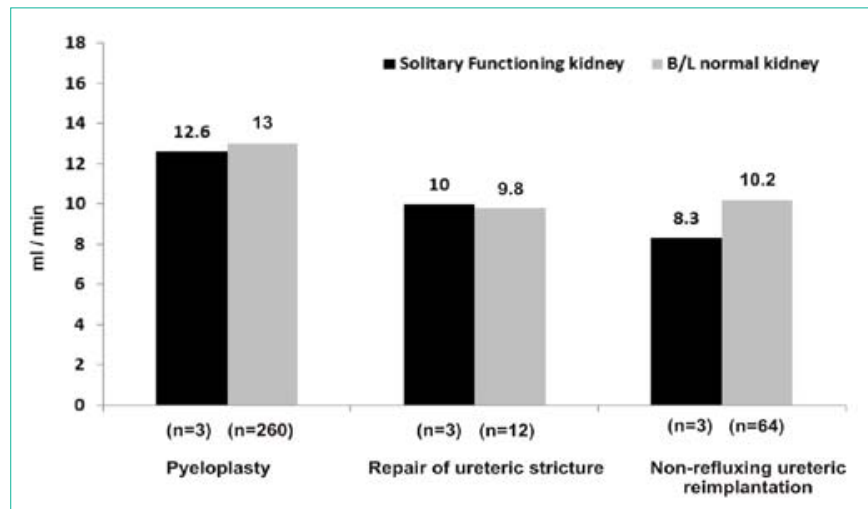

Figure 2: Comparison of improvement in GFR between 2 groups.

procedures were completed uneventfully with good outcome. Subsequently several reports have supported the feasibility and efficacy of this exercise in dealing with long segment lower ureteral pathologies [16-18]. However all these studies were carried out in patients with bilaterally functioning units. In our study in a group of 3 patients with benign ureteric stricture and SK/PSK, 2 patients presented with deranged renal function. Till last follow-up, one patient achieved normal renal profile and one patient is followed with improved renal function.

Open ureteric reimplantation has been considered the gold standard exercise for vesicoureteric reflux demanding surgical approach [19]. Recently laparoscopic and robot-assisted extravesical and intravesical surgeries have been attempted in view of the superior morbidity profile associated with these approaches. The reported early results following these exercises have been encouraging [2022]. In our cohort, 3 patients underwent laparoscopic antireflux construction in SK for high grade vesicouretral reflux (Grade III VUR in 1 patient and Grade IV VUR in 2 patients). The contralateral unit was extirpated in view of non-salvagability secondary to advanced reflux nephropathy. We prefer an extravesical approach universally as consequences secondary to breach of bladder mucosa may be avoided. Due to lack of availability no robotic assisted procedures were attempted. All our cases had resolution of symptoms with improved drainage profile. No reflux was remarkable in any patient in follow-up VCUG.

Laparoscopic reconstruction of obstructive megaureter is an emerging concept [23]. Although the procedure involves a multitude of tasks and is technically complex, in expert hands good results can be enjoyed. Our patient with obstructive megaureter presented with deranged renal profile pre procedure. He is asymptomatic till last follow-up with an improvement in renal profile.

Uniqueness of our study is that all reconstructive procedures were undertaken in solitary functioning kidney of which 4 patients also had impaired renal parameters pre procedure. All procedures could be successfully accomplished by laparoscopic approach. No patients required any additional intervention (stent or nephrostomy). Although last follow-up creatinine did not reach normal level in 2 patients, there was significant improvement in drainage pattern. Figure 2 depicts the comparison of improvement in GFR obtained at last follow-up in SK/PSK to that achieved following similar exercises in bilaterally functioning units (authors own series). No significant difference was perceived between the two groups. However the disparity in sample size hinders the derivation of a statistically validated inference.

Few important observations from our study need to be mentioned. The effect of pneumoperitoneum on renal function has always been debatable. In our cohort, a significant increment was recorded in the first postoperative day creatinine in comparison to preprocedure. This could be attributed to the effect of pneumoperitoneum on immediate postoperative creatinine level. However there was no impact on creatinine profile during revisits. Another aspect of these reconstructions needs to be emphasized. All procedures were undertaken by a single operator with more than 10 years of experience in laparoscopic surgeries. Additionally the operator embarked on these procedures only after gaining considerable experience in dealing with similar pathologies in bilateral functioning units. This exemplifies the complicacy of these procedures. Varsity with advanced laparoscopic exercises remains a bare necessity in handling these scenarios.

To the best of our knowledge there is no data available in literature till date regarding laparoscopic reconstruction of various upper urinary tract pathologies in presence of solitary kidney except for laparoscopic pyeloplasty. Limitations of our study are its retrospective design and small patient cohort. Larger prospective randomised studies with long term follow-up are required to justify the efficacy of laparoscopic reconstruction of various upper urinary tract pathologies in solitary or predominantly functioning kidney.

\section{Conclusion}

Laparoscopic reconstruction of upper urinary tract pathologies in solitary or predominantly solitary functioning kidney is feasible and safe. Although a significant elevation of renal profile may be encountered immediate post procedure, satisfactory improvement in renal function can be achieved at long term follow-up. Good results can be obtained in the hands of experienced laparoscopic urologist.

\section{References}

1. Permpongkosol S, Link RE, Su LM, Romero FR, Bagga HS, Pavlovich CP, et al. Complications of 2775 urological laparoscopic procedures: 1993 to 2005. J Urol. 2007; 177: 580-585.

2. Kaouk JH, Gill IS. Laparoscopic reconstructive urology. J Urol. 2003; 170: 1070-1078.

3. Rassweiler J, Pini G, Gozen AS, Klein J, Teber D. Role of laparoscopy in reconstructive surgery. Curr Opin Urol. 2010; 20: 471-482.

4. Symons SJ, Bhirud PS, Jain V, Shetty AS, Desai MR. Laparoscopic pyeloplasty: our new gold standard. J Endourol. 2009; 23: 463-467.

5. Moon DA, El-Shazly MA, Chang CM, Gianduzzo TR, Eden CG. Laparoscopic pyeloplasty: evolution of a new gold standard. Urology 2006; 67: 932-936.

6. Abraham GP, Das K, Ramaswami K, et al: Laparoscopic Boari flap ureteroneocystostomy in single and doule renal units: our experience. $\mathrm{J}$ of Endourol and part B Videourol. 2011.

7. Abraham GP, Das K, Ramaswami K, George DP, Abraham JJ, Thachill T, et al. Laparoscopic extravesical ureteric reimplantation for resolving vesicoureteric reflux in single, bilateral and duplex system-points of technique. J of Endourol and Part B, Videourol. 2011.

8. Abraham GP, Das K, Ramaswami K, George DP, Abraham JJ, Thachill T, et al. Laparoscopic dismemberment, excisional tailoring, reimplantation with antireflux- megamanagement for megaureter. J of Endourol and part B, Videourol. 2011. 
9. Knudsen BE, Cook AJ, Watterson JD, Beiko DT, Nott L, Razvi H, et al. Percutaneous antegrade endopyelotomy: Long-term results from one institution. Urology 2004; 63: 230-234

10. Szydełko T, Kopeć R, Kasprzak J, Apoznański W, Kołodziej A, Zdrojowy R, et al. Antegrade endopyelotomy versus laparoscopic pyeloplasty for primary ureteropelvic junction obstruction. J Laparoendosc Adv Surg Tech A. 2009; 19: 45-51.

11. Davenport K, Minervini A, Timoney AG, Keeley Jr FX. Our experience with retroperitoneal and transperitoneal laparoscopic pyeloplasty for pelvi-ureteric junction obstruction. Eur Urol. 2005; 48: 973-977.

12. Romero FR, Wagner AA, Trapp C, Permpongkosol S, Muntener M, Link RE, et al. Transmesenteric laparoscopic pyeloplasty. J Urol. 2006; 176: 25262529.

13. Albani JM, Desai MM, Gill IS, Streem SB. Repair of adult ureteropelvic junction obstruction in the solitary kidney: Effect on renal function. Urology 2006; 68: 718-722.

14. Zafar N, Leyland J, Shah NC. Laparoscopic pyeloplasty in a solitary kidney. Nat Clin Pract Urol 2007; 4: 625-629.

15. Fugita OE, Dinlenc C, Kavoussi L. The laparoscopic Boari flap. J Urol 2001 166: 51-53.

16. Simmons MN, Gill IS, Fergany AF, Kaouk JH, Desai MM. Laparoscopic ureteral reconstruction for benign stricture disease. Urology. 2007; 69: 280 284.
17. Rassweiler JJ, Gözen AS, Erdogru T, Sugiono M, Teber D. Ureteral reimplantation for management of ureteral strictures: A retrospective comparison of laparoscopic and open techniques. Eur Urol. 2007; 51: 512 522.

18. Castillo OA, Litvak JP, Kerkebe M, Olivares R, Urena RD. Early experience with the laparoscopic boari flap at a single institution. J Urol 2005; 173: 862 865.

19. Lakshmanan Y, Fung LC. Laparoscopic extravesicular ureteral reimplantation for vesicoureteral reflux: recent technical advances. J Endourol. 2000; 14 589-593.

20. Yeung CK, Sihoe JD, Borzi PA. Endoscopic cross-trigonal ureteral reimplantation under carbon dioxide bladder insufflation: a novel technique. $J$ Endourol. 2005; 19: 295-299.

21. Kutikov A, Guzzo TJ, Canter DJ, Casale P. Initial experience with laparoscopic transvesical ureteral reimplantation at the Children's Hospital of Philadelphia. J Urol. 2006; 176: 2222-2225.

22. Smith RP, Oliver JL, Peters CA. Pediatric robotic extravesical uretera reimplantation: comparison with open surgery. J Urol. 2011; 185: 1876-1881.

23. Agarwal MM, Singh SK, Agarwal S, Mavuduru R, Mandal AK. A Nove Technique of intracorporeal excisional tailoring of megaureter before laparoscopic ureteral reimplantation. Urology. 2010; 75: 96-99.
Austin J Clin Case Rep - Volume 4 Issue 2 - 2017

ISSN : 2381-912X | www.austinpublishinggroup.com

Das et al. (C) All rights are reserved
Citation: Das K, Abraham GP, Siddiaiah AT, Abraham JJ, Ramaswami K, Thampan OS, et al. Laparoscopic Reconstruction of Pathologies Involving Solitary/Predominantly Solitary Functioning Kidney-Feasibility, Safety and Outcome Analysis. Austin J Clin Case Rep. 2017; 4(2): 1120. 